\title{
Type I and Type II Micromachined Vibratory Gyroscopes
}

\author{
Andrei M. Shkel \\ Mechanical \& Aerospace Engineering \\ University of California - Irvine \\ 4208 Engineering Gateway Building \\ Irvine, CA, USA, 92697-3975 \\ E-mail: ashkel@uci.edu
}

\begin{abstract}
All Micromachined Vibratory Gyroscopes (MVG) are based on the principle that the Coriolis forces produced by rotation of the gyro cause a transfer of energy between two of the gyro's modes of vibration. Vibratory Gyroscopes can be classified into two broad types, angle gyroscopes (or Type I) and rate gyroscopes (or Type II). Gyroscopes of the first type measure orientation angles directly, while gyroscopes of the second type measure rotational rate. Most MVG implementations to date are found exclusively in the angular rate measuring variety, however there are opportunities for implementation on the micro-scale angle gyroscopes. This paper introduces a unified approach for description of MVGs and emphasizes the differences between the two types of devices. We also review our recent results in development of Type I and Type II gyroscopes.
\end{abstract}

\section{INTRODUCTION}

All vibratory gyroscopes use the Coriolis acceleration that arises in rotating reference frames to measure rotation. The Coriolis forces produced by vibration of the sensing element and rotation of the system cause a transfer of energy between two of the gyro's modes of vibration. Vibratory gyros divide naturally into two classes depending on whether the two modes are of the same kind or different. In the classical tuning fork gyro (Fig. 1), for example, the two modes are different. One consists of a flexural vibration of the tines and the other a torsional vibration about the stem. Vibratory gyros utilizing similar modes include the vibrating string, the vibrating rectangular bar, the vibrating cylinder, and the hemispherical resonator gyro, Fig. 2.

Vibratory gyros utilizing similar modes can be operated in the whole angle mode (direct angle measurement mode) or the open-loop mode(or force-to-rebalance modes) to measure rotational rate. Conventionally, different modes gyros are used for angular rate measurement. We introduced the classification of vibratory gyroscopes based on phenomena they measure angle gyroscopes (or Type I) and rate gyroscopes (or Type II).

Almost all reported to date Micromachined Vibratory Gyroscopes (MVG) are of Type II. They operate on the Coriolis principle of a vibrating proof mass suspended above the substrate. The proof mass is supported by anchored flexures, which serve as the flexible suspension between the proof mass and the substrate, making the mass free to oscillate in two orthogonal directions: the drive direction and the sense direction. In Type II implementation, the overall dynamical

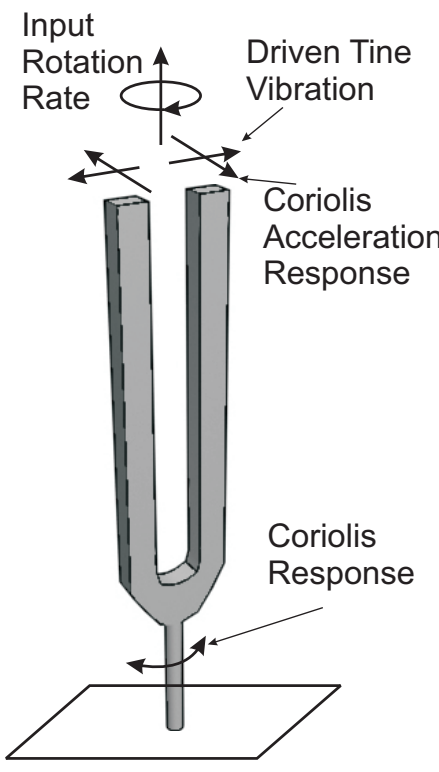

Fig. 1. Tuning Fork Gyroscope. Commonly used Type II gyro configuration

system is typically a two degrees-of-freedom (2-DOF) massspring-damper system with similar or different kind of modes, for example linear displacement drive and linear displacement sense, or linear displacement drive and angular displacement sense. In this type of gyroscopes the drive direction is typically excited by the electrostatic drive forces to a constant amplitude of oscillation (linear or angular depending on the design), and the sense direction is excited by the rotation-induced Coriolis force. Gyros depending on two different modes generally have large temperature sensitivity due to the differences in the temperature sensitivity of the mode natural frequencies. Gyros of inertial grade are therefore not typically sought in the Type II design configuration. It should be noted that this is by far the most common type of micromachined vibratory gyroscopes [11].

Type I gyroscopes are among the highest performance mechanical gyroscopes on the inertial market. They typically utilize symmetric vibratory shells or continuum isotropic structures, including vibrating string, the vibrating rectangular bar, the vibrating cylinder, or the hemispherical resonating shell, 


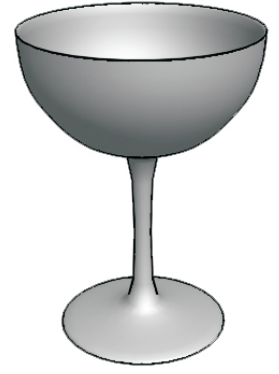

Wine-Glass

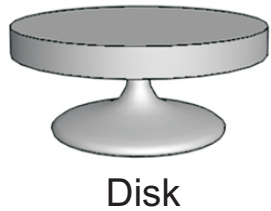

Fig. 2. Examples of vibrating shell gyroscopes

Fig. 2. The dynamic behavior of all the gyros of this type is equivalent to that of the Foucault pendulum. This analogy will be used in the discussion that follow. The two modes of the Foucault pendulum may be taken to be a pendulum motion (swing) in the north-south direction and a motion in the east-west direction. In the absence of rotation and damping, the pendulum swing will stay aligned with northsouth direction; and the stored energy will remain in the first normal mode indefinitely. Under rotation, however, the Coriolis acceleration will transfer energy between the two normal modes causing the pendulum to precess. The angle of precession is proportional to the angle of object rotation. The moment of inertia of the shell with respect to the axis aligned with the axis of rotation defines the coefficient of proportionality. If the Foucault pendulum is mechanically ideal and there are no sources of energy dissipation, it is sufficient to displace the pendulum from its equilibrium and it will be precessing indefinitely measuring the rotation. In the presence of non-idealities, which is always the case in reality, the main challenge of the control system is to compensate for asymmetries in the suspension system and asymmetries in damping, importantly without interfering with the Pendulum precession.

Of all vibratory gyroscopes of Type I considered in the last 60 years, only the vibrating cylinder and the hemishperical resonator demonstrated the potential for inertial grade performance. The implementation of Type I gyroscopes in the form of a shell is an interesting option to consider on the microscale, but does not seem to be currently feasible. The main reason is that microfabrication is typically limited to planar geometries and has limited fabrication tolerances. On the microscale we can build small planar structures, but we can only build those with limited precision. Manufacturing tolerances of lithography based techniques allow approximately $10^{-2}$ to $10^{-3}$ relative tolerances (the ratio between manufacturing imperfection and the minimum feature), which is several orders of magnitude larger than required for inertial grade performance gyroscopes. Additionally, 3-DOF high precision shells are difficult, if not impossible, to implement on the micro-scale. Even if we were able to implement precision micro-size shells, the number of sense electrodes and therefore nominal capacitance would be limited by the area of the shell.

Micromachined Type I gyroscopes are still an intriguing opportunity, fueled by the success of conventional size angle gyroscopes (the only type of compact mechanical gyroscopes which has achieved the inertial grade performance). These devices are used for inertial navigation.

The first idea for micro-scale version of Type I Micromachined Vibratory Gyroscope was first introduced in [1], Fig. 3(b).

The Type I MVG is also of the vibratory type and like Type II MVG utilizes the vibrating mass-spring-damper system, but in contrast to Type II is able to measure the angle of rotation directly, eliminating the need for numerical integration of the angular rate signal when information about the object orientation is required. Type I gyroscopes are two-dimensional isotropic resonators that are designed to transfer the energy between their principal axes of elasticity. In their operation, object rotation and free oscillations of an isotropic massspring system induce precession of the line of oscillation, with the angle of precession equal exactly to the angle of object rotation, [1]. The angle can be determined from the instantaneous position and velocity by the relationship given in Figure 3(a).

In commonly used Type II MVG, rotation and constant amplitude of oscillation of a mass-spring system along the drive axis induces response along the sense axis, Fig. 5(a). The angular velocity can be determined by measuring the steadystate deflection of the mass along the sense axes, Fig. 5(a). The conventional Type II gyroscopes are generally designed to operate at or near the peak of their resonance curve, which makes the system to be very sensitive to variations in system parameters. Two major design concepts explored by this group are reviewed in this paper, both allowing to achieve a dynamical system with a wide-bandwidth frequency response.

\section{DyNAMiCS OF VibRATORY GYROSCOPES}

Simplified dynamic equation of motion of a broad class of Micromachined Vibratory Gyroscopes can be described in non-inertial coordinate frame as

$$
\begin{aligned}
& m \ddot{x}=-c_{x} \dot{x}-k_{x} x+m\left(\Omega^{2} x+2 \Omega \dot{y}+\dot{\Omega} y\right)+F_{x} \\
& m \ddot{y}=-c_{x} \dot{y}-k_{y} y+m\left(\Omega^{2} y-2 \Omega \dot{x}-\dot{\Omega} x\right)+F_{y}
\end{aligned}
$$

where $\Omega$ is the angular velocity of the coordinate frame associated with the gyroscope, $m$ is the mass, $c_{x}$ and $c_{y}$ are damping coefficients, $k_{x}$ and $k_{y}$ are stiffness coefficients, and $F_{x}$ and $F_{y}$ are additional externally applied forces to the mass with respect to the reference coordinate frame.

Gyroscopes are typically designed for detection of small angular velocities, and often the constant rate conditions $(\dot{\Omega}$ 
= 0) can be assumed (if this is not the case, the control electronics is designed to compensate for the corresponding inertial force). Since the natural frequencies of gyroscopes are typically in the range of $1 \times 10^{4}$ to $1 \times 10^{5} \mathrm{rad} / \mathrm{sec}$ and the measured rotational rates are typically on the order of $1 \mathrm{rad} / \mathrm{sec}, \omega_{x}^{2} \gg \Omega^{2}$ and $\omega_{y}^{2} \gg \Omega^{2}$. These assumptions allow to ignore terms with $\Omega^{2}$ and $\dot{\Omega}$ in the equation of motion. Additionally, most gyroscopes are designed such that the two natural frequencies and damping ratios are ideally identical $\left(\omega_{x}=\omega_{y}=\omega_{n}, \zeta_{x}=\zeta_{y}=\zeta\right)$. Based off these typical operational considerations, the governing equation of motion of MVG can be simplified to

$$
\begin{aligned}
& \ddot{x}+\frac{\omega_{n}}{Q} \dot{x}+\omega_{n}^{2} x-2 \Omega \dot{y}=\frac{F_{x}}{m} \\
& \ddot{y}+\frac{\omega_{n}}{Q} \dot{y}+\omega_{n}^{2} y+2 \Omega \dot{x}=\frac{F_{y}}{m}
\end{aligned}
$$

where $Q$ is the quality factor, which is defined as the ratio of energy stored per oscillation cycle to energy lost per oscillation cycle $(Q=1 / 2 \zeta)$. In a basic formulation, Equation (2) describes the ideal dynamics of a typical vibratory gyroscope.

\section{TYPE I GYROSCOPES}

A Type I gyroscope is defined as a gyroscope which measures absolute angles of rotation. The ideal operation of a Type I gyroscope requires the free oscillation of the mass. In order to fulfill this criteria, it is necessary to utilize an energy sustaining feedback control which cancels the effects of damping. Such control architectures are discussed in [3], [9], [5]. Under this assumption of a zero damping condition, the only perturbations are Coriolis force. If an isotropic oscillator is displaced from their position of equilibrium, then for an observer in the rotating frame, due to the Coriolis force, the energy of vibration will be transferred between the two principal axes of elasticity. Equivalently, the line of oscillation of the resonator will precess in the non-inertial coordinate frame. This phenomena is illustrated in Figure 3. It can can be shown that the orientation $\phi$ of the elliptical trajectory of the mass remains constant in inertial space by the fact that $\phi$ exactly follows the negative angle of rotation

$$
\phi=-\int \Omega d t
$$

The orientation angle $\phi$ can be directly determined from the instantaneous position and velocity by the relationship

$$
\tan 2 \phi=\frac{2\left(\omega_{n}^{2} x y+\dot{x} \dot{y}\right)}{\omega_{n}^{2}\left(x^{2}-y^{2}\right)+\left(\dot{x}^{2}-\dot{y}^{2}\right)}
$$

Alternatively, the angle of precession can be determined by measuring displacement of the mass along two orthogonal directions.

One of the most successful vibratory gyroscopes on the inertial navigation market is the Hemispherical Resonance Gyroscopes (HRG). This device is operating on the principle of elastic waive precession described above. The sensing

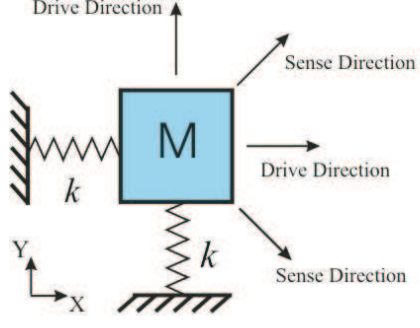

Equation of Motion

$$
m \ddot{x}+k x+2 m \Omega \dot{y}=0
$$$$
m \ddot{y}+k y-2 m \Omega \dot{x}=0
$$

\section{(a)}

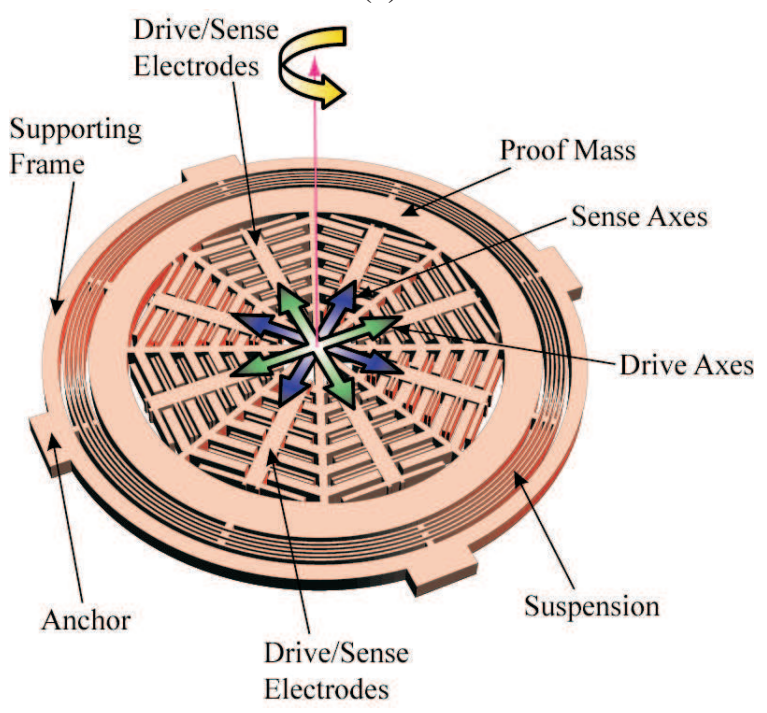

(b)

Fig. 3. (a) Ideal Response of Type I gyroscope; (b) A design of Type I gyroscope, [1]

element of HRG is a precision shell made out of quartz. Even though desirable, it is not feasible to implement a shell on the micro-scale with required precision for Type I operation. In [1], we proposed a non-shell implementation of Type I gyroscope, and thus made it feasible to implement the concept on the micro-scale. It was demonstrated that an isotopic 2-DOF resonator has the same dynamics as the isotropic symmetric shell and thus can be used as Type I, or angle measuring gyroscope. There is a fundamental difference between the shell and the isotropic resonator behavior. In the case of a shell a standing waive is excited in the shell and precession of the elastic waive is detected and related to the object rotation. In the case of the resonator, however, the standing waive notion is not appropriate and is replaced by vibration pattern of the resonator itself and the transfer of energy, or precession of the vibration pattern, is used for detection of the object rotation.

The modes of any realistic gyroscope will, in general, have slightly different natural frequencies due to asymmetries in the suspension system. Because of the differences of the natural frequencies along the principle axes of elasticity, an initially 
planar pendulum swing (or proof mass oscillation, in the case of the isotropic oscillator) in the northeast-southwest direction will become elliptical with a growing minor axis amplitude of the pendulum (or resonator) orbit. To insure that the pendulum swing plane remains stationary with respect to to inertial space, it is not adequate to adjust the suspension to match the natural frequencies to a higher degree of accuracy. Because the transfer of energy between the modes caused by the Cariolis forces is highly sensitive to the relative phase of the normal mode components, these components must be maintained in phase by a closed-loop control system. The signals from the pickoffs monitoring the resonator motion must be processed to identify the ellipticity of the orbit (or quadrature). An error signal proportional to the ellipticity must then be fed back to forces to drive the ellipticity of the orbit to null.

When the orbit ellipticity control loop is functioning, the principal source of drift is asymmetric damping. There will invariably be one direction of pendulum swing (or mass vibration) in which the pendulum motion is damped least. If the forcer system that supplies energy to replenish that lost to damping is symmetric, a pendulum initially swinging in any other direction will end up swinging in this preferred direction (at least in the absence of an Earth rate component input). This case-oriented drift rate as a function of pendulum direction must be stable enough to be calibrated (together with its temperature dependance) and compensated in control electronics algorithms. Thus, the control architecture of Type I gyroscopes should allow the device freely precess in response to the angular rotation, while compensating for quadrature errors and asymmetric damping.

\section{A. Design}

The preferable MEMS implementation of Type I gyroscope is an isotropic oscillator, similar to the one shown in Fig. 3(b). The Type I MVGs generally consist of a mass connected to a suspension system, which is then in turn rigidly connected to a substrate below via anchors. Under ideal design, the suspension has isotropic stiffness with identical principal axes of elasticity, providing the same stiffness in all directions. The device is actuated electrostatically using fixed parallel plate electrodes anchored to the substrate below and interwoven throughout the mass.

Type I devices are fundamentally free oscillating 2 dimensional resonators, when a shell structure is utilized the equivalent equation of motion is obtained by replacing the continues shell equation of deformation with the corresponding nodal equation. To detect the precession (or energy transfer), the detection scheme is designed to measure both position and velocity of the mass within a two dimensional operating plane. In our implementation, the Type I sensing scheme uses transimpedance configuration for velocity detection and an additional cascaded integrator for position detection. The control architecture for the z-axis gyroscope uses two orthogonal pairs of parallel plate drive electrodes. The orthogonal pairs of drive and sense electrodes are oriented with $45^{\circ}$ shift, Figure 4(a). In order to obtain the true positions and velocities, the outputs from the sense electrodes is transformed by a $45^{0}$ rotational transformation. The control electronics includes the feedforward and feedback controls for anisoelasticity compensation and two-dimensional damping feedback compensating control. For details on control implementation please see [5].

\section{B. Results}

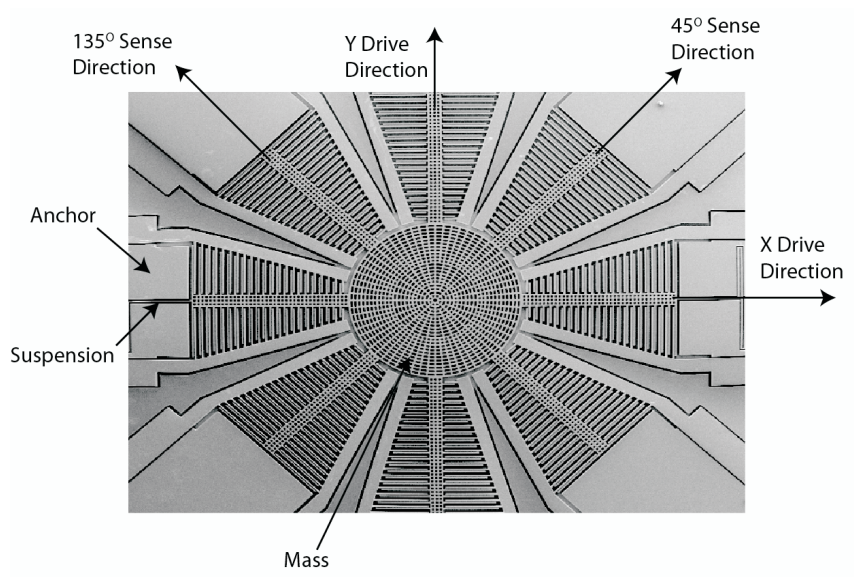

(a)

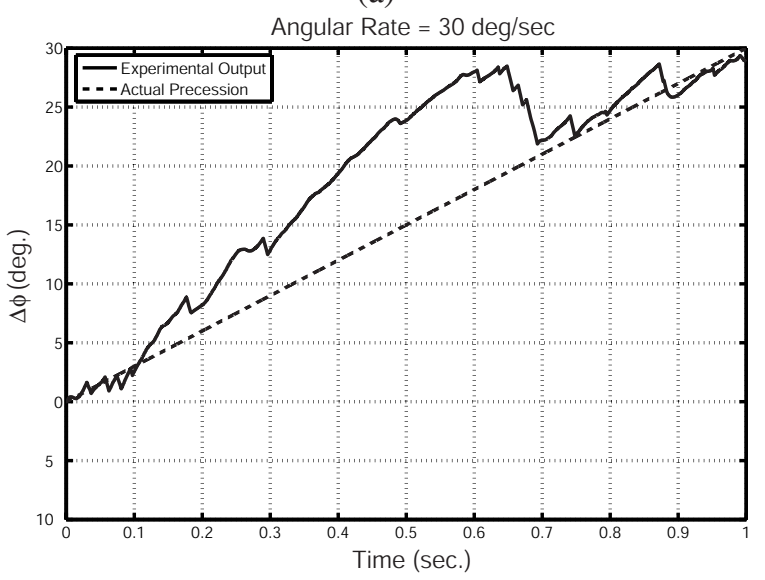

(b)

Fig. 4. (a) SEM image of a Type I gyroscope; (b) Measured precession of a Type I gyroscope [5]

A family of Type I prototypes has been implemented using SOI Bulk micromachining technology and characterized on a rate table, Figure 4(a). The devices were initially driven in an open loop mode using an AC voltage along $0^{0}$ and $45^{0}$ directions. In Figure 4(b), the angular rate of $30^{\circ} / \mathrm{sec}$ was applied and response of the device was measured using parallel plates electrostatic detection method. In the rate table experiments, the fabricated device demonstrated rate integrating properties, responding proportionally to the angle of rotation. The observed overshooting of the precession angle is explained by step-changes in angular velocity and uncompensated frequency splits between the primary axes of elasticity. The largest contribution to the unstable nature of the precession pattern is the phase stability of the control loop. This is the first experimental demonstration of Type I MVG 
operation. The comprehensive coverage of issues related to Type I MVG can be found in [5].

\section{TYPE II GYROSCOPES}

\section{A. Principle}

A Type II gyroscope is defined as a gyroscope which measures rate of angular rotation. These devices are designed with a defined "drive" axis along which the device is continually driven using applied force $F_{x}$ and a defined "sense" axis orthogonal to the drive axis where Coriolis induced motion is sensed, Figure 5(a). In this discussion, $x$-axis is defined to be the drive axis and $y$-axis is defined to be the sense axis. Type II gyroscopes are driven in either open loop mode, where the applied force is a state independent sinusoidal input $F_{x} / m=V_{D} \sin \omega_{n} t$, or in closed loop mode, where a feedback system maintains a constant amplitude of oscillation along the drive direction, [10].

In the case of the closed loop control of the drive mode oscillation, assuming external force $F_{y}$ is zero along the sense direction, substitution of a constant drive amplitude $x(t)=X_{D} \sin \omega_{n} t$ into the $Y$ motional dynamics of Equation (2) gives the Coriolis force induced output steady state $y$ deflection amplitude as

$$
|y(t)|=\frac{2 X_{D} \Omega Q}{\omega_{n}}
$$

This shows that the output sense deflection is proportional to the input angular rate.

In many of the applications being considered for miniature gyroscopes the open-loop response time may be too slow. For these applications, vibratory gyroscopes of Type II are operated in the force-to-rebalance, or closed-loop mode. In this mode of operation the gyroscope is driven to a fixed amplitude in the "drive" mode along the x-axis. Only now, the secondary mode amplitude is continuously monitored and driven to zero by applying the appropriate drive force along the y-axis, the "sense". Effectively then, a force applied along the y-axis to rebalance the Coriolis force so that no net force is applied to the sense direction. As a result the bandwidth of the sensor can be increased to values approaching the resonant frequency by the closed-loop electronics. However, there is a penalty for increasing the bandwidth because the signal-to-noise ratio also increases. This same engineering trade-off occurs in many sensors including some accelerometers. It can be shown that in the force-to-rebalance mode of operation, the sense mode drive force to the primary mode drive force is given by

$$
\left|\frac{F_{y}}{F_{x}}\right|=2 Q \frac{\Omega}{\omega_{n}}
$$

Note that this is the same relationship between the ratio of sense/drive amplitudes for the open-loop mode of operation. Rotation rate then can be obtained by measuring the secondary mode drive force when the pendulum is operated in the forceto-rebalance mode.

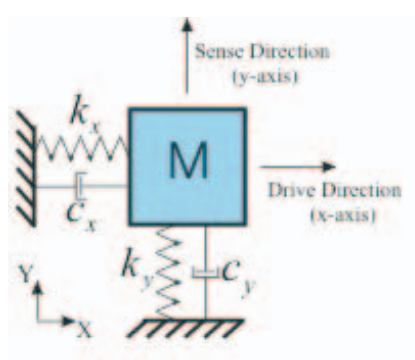

Equation of Motion

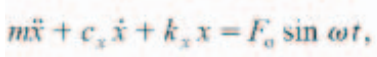

$m \ddot{y}+c_{y} \dot{y}-2 m \Omega \dot{x}+k_{y} y=0$

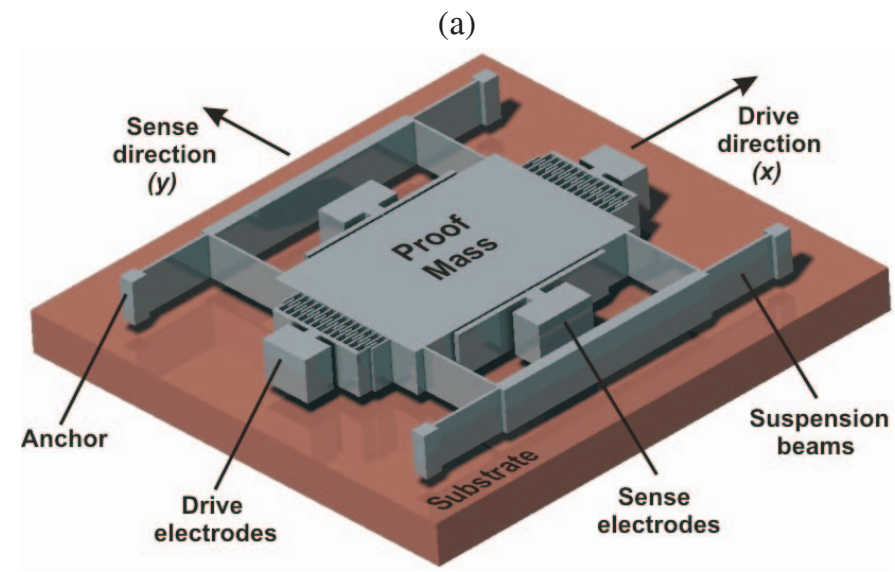

(b)

Fig. 5. (a) Theoretical Response of a classical Type II gyroscope; (b) A Design of Type II gyroscope

\section{B. Design}

The drive and sense mode matching requirement in vibratory gyroscopes renders the system response very sensitive to variations in system parameters due to fabrication imperfections and fluctuations in operating conditions, which shift the drive or sense resonant frequencies. Fabrication tolerance often results in natural frequencies that are mismatched by a few percent.

For the devices packaged in vacuum to enhance the sensitivity, the bandwidth of the resonance peaks is extremely narrow; leading to much tighter mode-matching requirements. Extensive research has been focused on design of symmetric drive and sense-mode suspensions for mode-matching and minimizing temperature dependence. However, especially for lightly-damped devices, the mode-matching requirement is well beyond fabrication tolerances; and none of the symmetric designs can provide the required degree of mode-matching without feedback control.

Over the last several years the research group at the University of California at Irvine introduced and experimentally demonstrated several new classes of MEMS rate gyroscope design, where the inherent robustness against structural and environmental parameter variation was achieved structurally, 


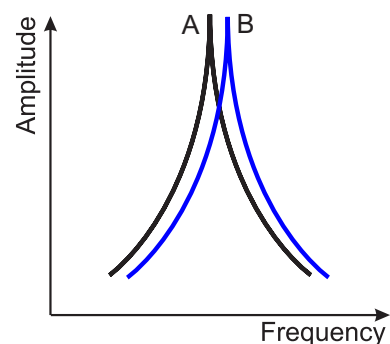

(a)

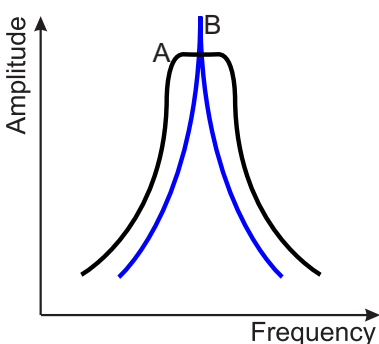

(b)
Fig. 6. (a) Dynamic response of a conventional 2-DOF vibratory gyroscope in the drive and sense directions; (b) Desirable dynamic response of a vibratory gyroscope in the drive and sense directions

effectively shifting the complexity from the control electronics to the structural design of gyroscope dynamical system.

The general approach is to explore the possibility of enhance robustness by achieving wide-bandwidth frequency responses in the drive and sense-modes. The conventional gyroscopes are generally designed to operate at or near the peak of their resonance curve. This is typically achieved by matching drive (A) and sense (B) resonant frequencies, Figure 6(a). However, the system is very sensitive to variations in system parameters due to fabrication imperfections and fluctuations operating conditions, which shift the drive or sense resonant frequencies. Under high quality factor conditions the gain is high, however, the bandwidth is extremely narrow. The desirable dynamic response of a vibratory gyroscope in the drive and sense direction is shown in Figure 6(b).

Two major design concepts were explored by our group in order to achieve a dynamical system with a wide-bandwidth frequency response: expanding the system design space by (1) increasing the degrees-of-freedom (DOF) of the sense mode oscillatory systems, [7], and (2) utilizing multiple drive-mode oscillators with incrementally spaced resonant frequencies [4], [2], [8].

1) Non-Resonant Vibratory Gyroscopes: One approach to increase bandwidth of a vibratory rate gyroscope is to design non-resonant multi-degree of freedom resonator, for example utilizing a 3-DOF dynamic system, as opposed to the 2-DOF system conventionally used in Type II MEMS gyroscopes. The 3-DOF design concept aims to utilize resonance in either the drive mode or the sense mode to improve sensitivity while maintaining the robust operational characteristics. This is achieved by forming, for example, structurally decoupled 1-DOF and 2-DOF oscillators in the drive and sense-modes, Fig. 7. The 2-DOF oscillator frequency response has two resonance peaks, and a flat region between the peaks, that defines the operation frequency region, Fig. 8. The 1-DOF drive oscillator resonant frequency is designed to overlap with the sense-mode flat operating region. Thus, the device is operated at the resonant frequency of the 1-DOF oscillator, while 2-DOF oscillator amplitude is inherently constant within the same frequency band. The comprehensive study of design options for multi-degree of freedom gyroscopes can be found in [4]

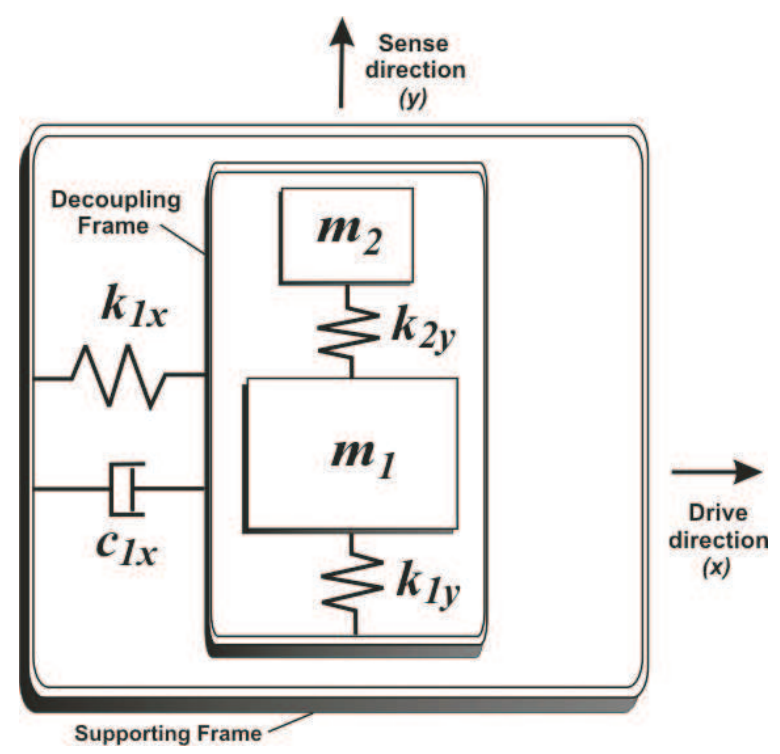

Fig. 7. Lumped mass-spring-damper model of the overall 3-DOF gyroscope dynamical system with 1-DOF drive-mode and 2-DOF sense-mode

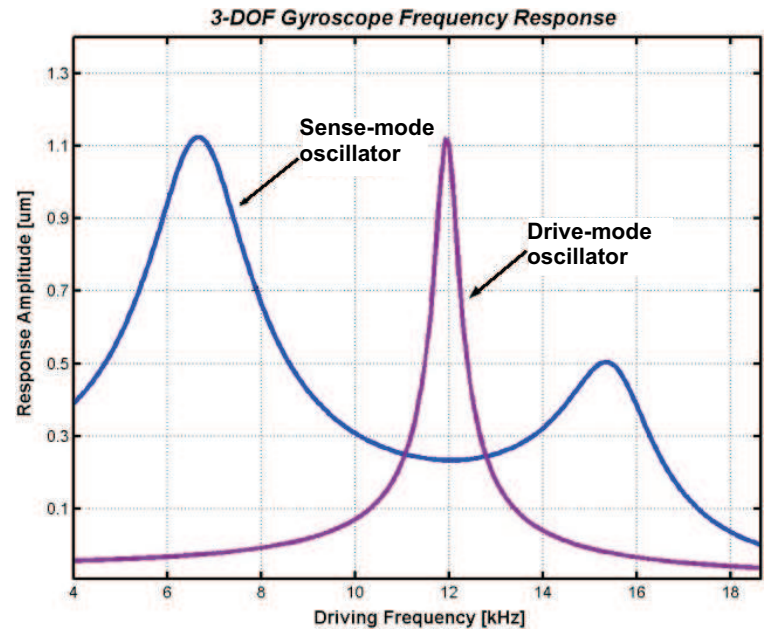

Fig. 8. The frequency responses of the 1-DOF drive and 2-DOF sensemode oscillators. The sense-direction oscillation amplitude is insensitive to parameter variations and damping fluctuations in the flat operating region, eliminating mode-matching requirement

The detection and control electronics for Type II MVGs is generally simpler than for Type I MVGs, as the drive and response detection are fundamentally one dimensional. The sense and drive electronics for Type II devices are extensively discussed in the literature and is not covered here.

2) Distributed Mass Vibratory Gyroscopes: The design concept is based on forming multiple drive-mode oscillators, distributed symmetrically around the center of a supporting frame [6]. The distributed drive-mode oscillators are driven in-phase towards the center of symmetry, and are structurally constrained in the tangential direction with respect to the supporting frame. Each oscillator is driven at the same drive frequency. In the presence of an angular rotation rate about the z-axis, a sinusoidal Coriolis force at the drive frequency is 


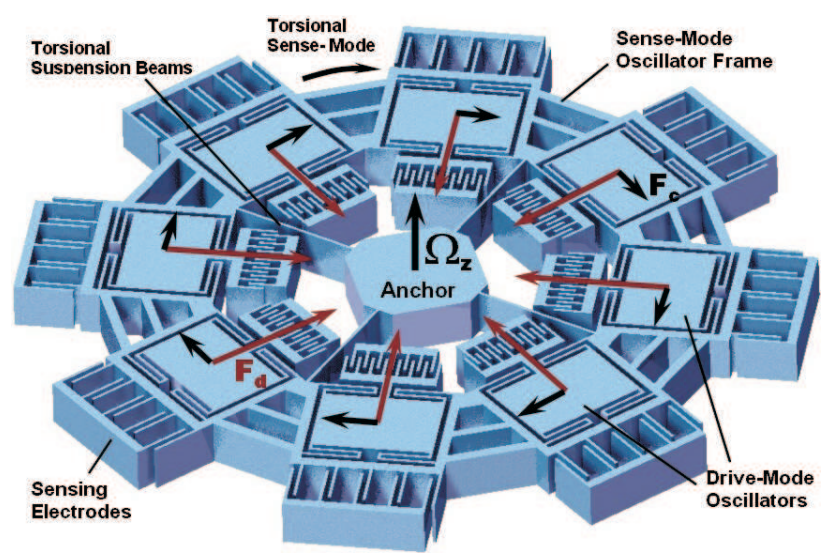

Fig. 9. Conceptual illustration of the Distributed-Mass Gyroscope with 8 symmetric drive-mode oscillators

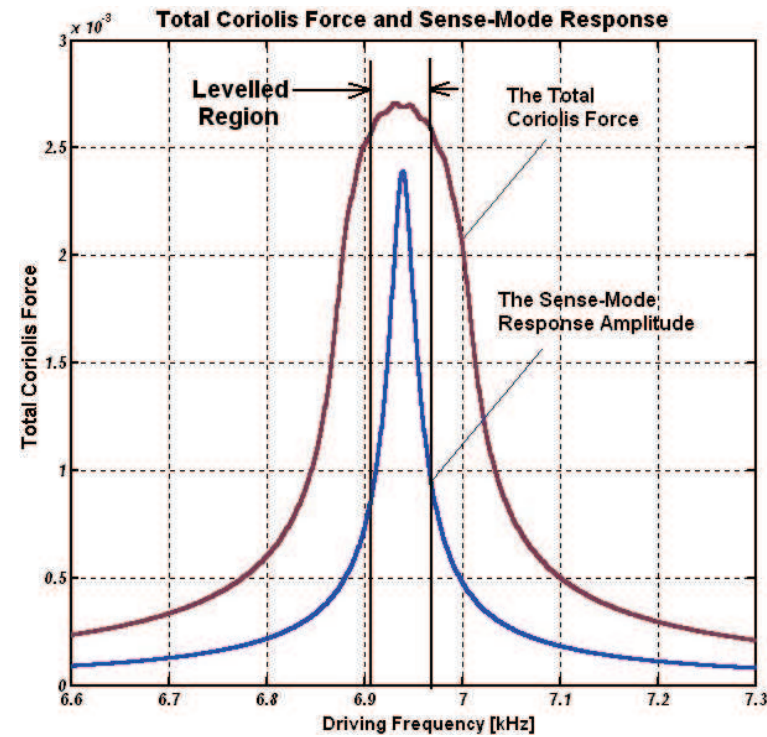

Fig. 10. The frequency spectrum of the total Coriolis torque generated by the distributed drive-mode oscillators

induced on each proof mass in the direction orthogonal to each drive-mode oscillation directions (Figure 9). Thus, each of the induced Coriolis force vectors lie in the tangential direction, combining to generate a resultant torque on the supporting frame. The net Coriolis torque excites the supporting frame into torsional oscillations about the $\mathrm{z}$-axis, which are detected by sense capacitors for angular rate measurement.

In the presented design concept, a wide-bandwidth operation region is achieved in the drive-mode frequency response, by designing or actively tuning the resonance frequency of each drive-mode oscillator to be incrementally spaced. Since the tangential Coriolis forces induced on each proof mass jointly generate a resultant torque on the supporting frame, a "levelled" total Coriolis torque is achieved over a wide range of driving frequencies. The device is nominally operated in this levelled region of the Coriolis torque frequency response, so that fluctuations in system parameters that shift oscillator resonance frequencies will not result in a significant change in the total Coriolis torque. If the sense-mode resonance frequency is designed to be accommodated in the same frequency band (Figure 10), the requirement on the degree of modematching is relaxed, and robustness against structural and thermal parameter fluctuations is achieved.

This design approach provides wider drive-mode bandwidth in vibratory MEMS gyroscopes. The approach was theoretically illustrated, and experimentally verified in [6].

\section{Results}

In this section the Non-Resonant Type II MVG is illustrated. The synchronous demodulation technique was utilized to extract the angular rate response of the 3-DOF system at different temperatures. The device with 1-DOF drive and 2DOF sense was used for this demonstration. The drive signal applied on the comb-drive actuators was $25 \mathrm{~V}$ DC bias and $3 \mathrm{~V}$ AC. The drive-mode amplitude was continuously monitored during the operation of the device using a microscope attached to the rate-table platform. The temperature of the device also continuously monitored using a solid-state temperature sensor attached to the sample.

A lock-in amplifier was utilized to provide the driving AC signal applied on the comb-drives, and to synchronously demodulate the Coriolis signal at the drive frequency in the internal reference mode. The output of the lock-in amplifier is low-pass filtered to provide a stable output reading proportional to the applied input angular rate.

When the temperature of the gyroscope was increased from $25^{\circ} \mathrm{C}$ to $75^{\circ} \mathrm{C}$, while keeping the excitation frequency constant at $752 \mathrm{~Hz}$, the sensitivity of the gyroscope was observed to drop from $0.0308 \mathrm{mV} /{ }^{0} / \mathrm{s}$ to $0.0234 \mathrm{mV} /{ }^{0} / \mathrm{s}$ (Figure $11(\mathrm{~b})$ ). This translates into $24.1 \%$ drop in the response gain. When the change in the drive-mode amplitude from $25^{\circ} \mathrm{C}$ to $75^{\circ} \mathrm{C}$ is investigated, it is seen that it changes from $5.8 \mu \mathrm{m}$ to $4.3 \mu \mathrm{m}$; yielding a $25.9 \%$ change. Thus, it is demonstrated that the change in the gyroscope sensitivity is almost exactly equal to the drive-mode amplitude change (with 1.8\% discrepancy); verifying the insensitivity of the sense-mode response to temperature variations.

In order to compare the improved robustness of the discussed wide-bandwidth approach, a micromachined gyroscope with a conventional 2-DOF dynamical system was characterized under the same temperature variations and using the same signal conditioning electronics.

When the temperature of the tested conventional gyroscope was increased from $25^{\circ} \mathrm{C}$ to $75^{\circ} \mathrm{C}$ while restoring the drivemode amplitude to $12 \mu \mathrm{m}$, the sensitivity was observed to drop from $0.91 \mathrm{mV} /{ }^{\circ} / \mathrm{s}$ to $0.73 \mathrm{mV} /{ }^{\circ} / \mathrm{s}$. Thus, a $50^{\circ} \mathrm{C}$ temperature increase was observed to result in $19.8 \%$ sensitivity change in the conventional gyroscope, which is over 12.2 times larger than the wide-bandwidth gyroscope approach.

The presented experimental results illustrate that the idea of the wide-band distributed mass Type II gyroscopes is feasible. The unique characteristics of the design idea may lead to a class of MEMS gyroscopes with the order of magnitude 


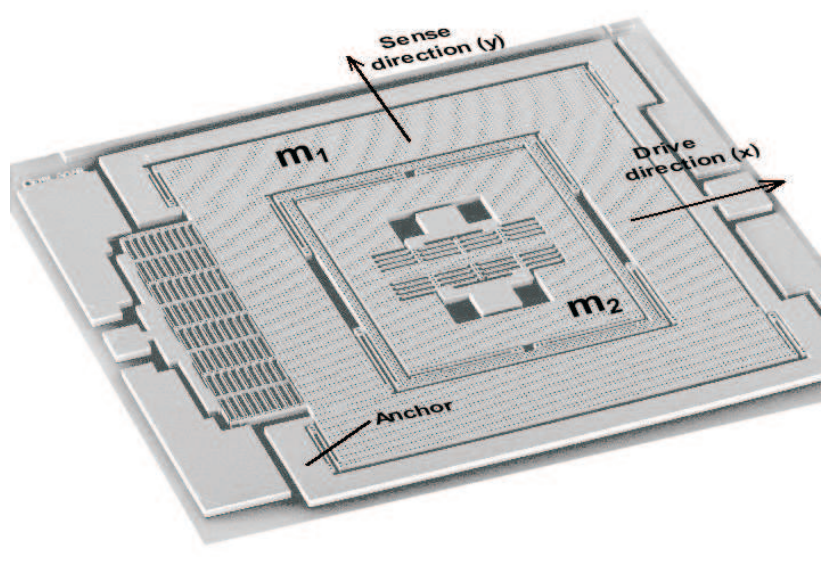

(a)

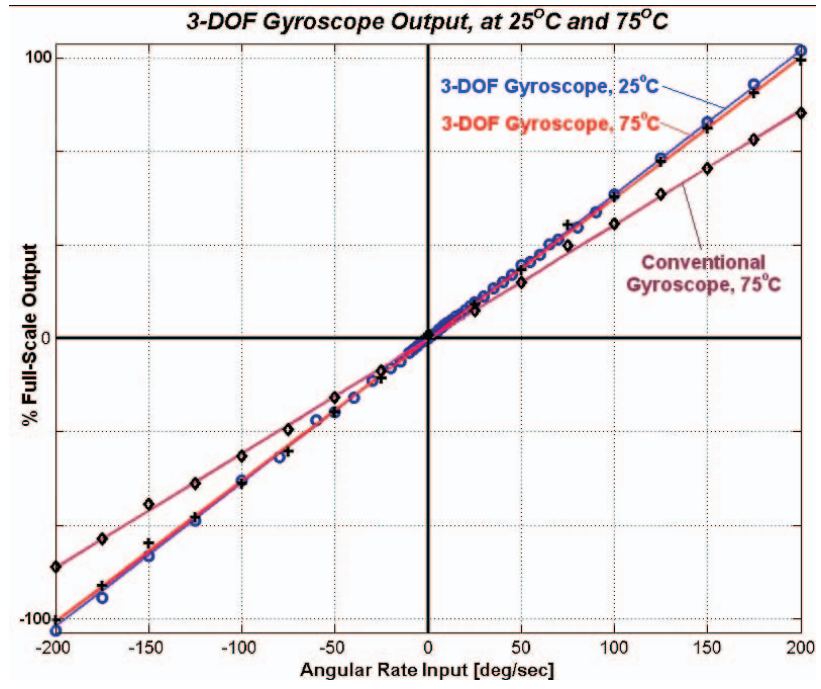

(b)

Fig. 11. (a) SEM image of non-resonant Type II gyroscope; (b) The angularrate response of the 3-DOF gyroscope with 2-DOF sense-mode, at $25^{\circ} \mathrm{C}$ and $75^{\circ} \mathrm{C}$.

increase in the bandwidth and robustness without sacrifice in sensitivity.

\section{Conclusions}

We introduced and demonstrated Type I and Type II Micromachined Vibratory Gyroscopes.

For the first time, a Type I MVG has been realized and demonstrated. The devise was verified to be capable of measuring absolute angles of rotation directly, [3]. The results are preliminary, and future work will focus on understanding the factors effecting performance of Type I gyros. For comprehensive coverage of this topic please see [5].

We summarized two approaches for designing widebandwidth Type II Micromachined Vibratory Gyroscopes. This topic was extensively studied in [4]. The demonstrated 3DOF prototypes exhibited a noise-floor of $0.640 / s / \sqrt{H z}$ in atmospheric pressure, with the designed off-chip detection electronics based on synchronous demodulation. Rate table experiments at $25^{\circ} \mathrm{C}$ and $75^{0} \mathrm{C}$ showed only $1.62 \%$ change in the scale factor without active control, while conventional devices resulted in $19.8 \%$ scale factor change, verifying the improved robustness of the non-resonant Type II MVG design concept.

The micromachined gyroscopes of both types could potentially yield reliable, robust and high performance angularrate measurements leading to a wide range of applications including dynamic vehicle control, automotive safety systems, navigation/guidance systems, and interactive consumer electronics.

\section{ACKNOWLEDGMENT}

This work was partially supported by the National Science Foundation Grants CMS-0409923 and CMS-0449442, program director Dr. Shih-Chi Liu. This work was also cosponsored by BEI Technologies Inc. and the University of California Discovery Program. The topics discussed in this review paper were extensively studied in two recently defended Ph.D. thesis by Chris Painter [5] on Type I MVG and by Cenk Acar [4] on Type II MVG.

\section{REFERENCES}

[1] A. M. Shkel and R.T. Howe "Surface Micromachined Rate Integrating Gyroscopes" US Patent No. 6,481,285

[2] C. Acar and A. M. Shkel "Non-Resonant Four Degrees-of-Freedom Micromachined Gyroscope" U.S. Patent No. 6,845,669

[3] C. Painter and A. M. Shkel "Multi Stage Control Architecture for Error Supression in Micromachined Gyroscopes" U.S. Patent No. 6,934,660

[4] C. Acar "Robust Micromachined Vibratory Gyroscopes" Ph.D. thesis, Dept. of MAE, University of California - Irvine 2004

[5] C. Painter "Micromachined Vibratory Gyroscopes with Imperfections" Ph.D. thesis, Dept. of MAE, University of California - Irvine 2005

[6] C. Acar and A. M. Shkel "An Approach for Increasing Drive-Mode Bandwidth of MEMS Vibratory Gyroscopes" Journal of Microelectromechanical Systems Vol. 14, No. 3, pp. 520-528, 2005

[7] C. Acar and A. M. Shkel "Inherently Robust Micromachined Gyroscopes With 2 DOF Sense-Mode Oscillator" Journal of Microelectromechanical Systems Vol. 15, No. 2, pp. 380-387, 2006

[8] C. Acar and A. M. Shkel "Non-Resonant Micromachined Gyroscopes with Mode-Decoupling" IEEE Sensors Journal vol. 3, No. 4, August 2003, pp. 497-506

[9] C. Painter and A. M. Shkel "Active Structural Error Supression in MEMS Vibratory Rate Integrating Gyroscopes" IEEE Sensors Journal Vol. 3, No.5, pp. 595-606, 2003

[10] N. Yazdi, F. Ayazi,and K. Najafi "Micromachined Inertial Sensors" Proceedings of the IEEE, volume 86, Number 8 pp. 1641-1659, August, 1998

[11] J. Soderkvist "Micromachined gyroscopes" Sensors and Actuators A volume 43, pp.65-71, 1994 